\title{
Active optics challenges of a thirty- meter segmented mirror telescopy
}

George Z. Angeli, Robert S. Upton, Anna Segurson, Brent L. Ellerbroek

George Z. Angeli, Robert S. Upton, Anna Segurson, Brent L. Ellerbroek, "Active optics challenges of a thirty-meter segmented mirror telescopy," Proc. SPIE 5382, Second Backaskog Workshop on Extremely Large Telescopes, (7 July 2004); doi: 10.1117/12.566326

SPIE Event: Second Backaskog Workshop on Extremely Large Telescopes, 2003, Backaskog, Sweden 


\title{
Active optics challenges of a thirty meter segmented mirror telescope
}

\author{
George Z. Angeli ${ }^{l}$, Robert Upton ${ }^{l}$, Anna Segurson ${ }^{\prime}$, Brent Ellerbroek ${ }^{l}$ \\ ${ }^{I}$ New Initiatives Office, AURA Inc.
}

\begin{abstract}
Ground-based telescopes operate in a turbulent atmosphere that affects the optical path across the aperture by changing both the mirror positions (wind seeing) and the air refraction index in the light path (atmospheric seeing). In wide field observations, when adaptive optics is not feasible, active optics are the only means of minimizing the effects of wind buffeting. An integrated, dynamic model of wind buffeting, telescope structure, and optical performance was developed to investigate wind energy propagation into primary mirror modes and secondary mirror rigid body motion. Although the results showed that the current level of wind modeling was not appropriate to decisively settle the need for optical feedback loops in active optics, the simulations strongly indicated the capability of a limited bandwidth edge sensor loop to maintain the continuity of the primary mirror inside the preliminary error budget. It was also found that the largest contributor to the wind seeing is image jitter, i.e. OPD tip/tilt.
\end{abstract}

Keywords: active optics, telescope control, segmented mirror control, wind buffeting

\section{INTRODUCTION}

One of the major challenges the design of a 30 meter class optical telescope encounters is the mitigation of the wind induced deformations and vibrations of the optical surfaces. Although the full extent of this problem is not yet completely understood, there are indications ${ }^{1}$ that wind buffeting is going to be more severe than for the current 10 meter class telescopes. This is due to the larger cross section of the telescope and the larger mass of the telescope, which implies structural resonances closer to the frequency range where the wind carries most of its energy.

Some of the science goals associated with the proposed extremely large telescopes imply wide field observations (up to 30 arcmin). Since adaptive optics is not yet feasible for such a large field of view, these observations are constrained by the atmospheric seeing. One of the major objectives of the active optics to be implemented is to avoid additional limitations due to wind induced image aberrations, i.e. wind seeing. The other critical issue is the continuity of the wavefront produced by the telescope. Discontinuities not only destroy the image quality, but also prevent accurate wavefront sensing essential to adaptive optics corrections.

There are well-developed techniques for controlling the continuity of large segmented primary mirrors ${ }^{2,3}$. Preliminary studies show, that even for extremely large number of segments (up to 1080), actuator and sensor noise propagation issues are not preventing these techniques from keeping the continuity of the mirror inside the error budget ${ }^{4}$. Simulations using real wind pressure measurements indicate that the control technique used on 10 meter segmented mirror telescopes has the potential to mitigate the effect of wind buffeting on the primary mirror segments for 30 meter telescopes, as far as the continuity of the mirror is concerned ${ }^{5}$. However, the necessary control bandwidth is an open question that can be answered only by means of dynamic, integrated simulations of the telescope structure, wind loading and control systems. A possible control bandwidth overlapping with the telescope structural resonances has far reaching implications on the control laws required and the difficulties the control design encounters.

It is also well known that the edge sensors used to maintain the continuity of the segmented mirror are quite insensitive to low order shape deformations of the whole mirror ${ }^{6}$. Although the extent of this lack of sensitivity depends on the actual

"gangeli@gemini.edu; phone 520-318-8413; AURA New Initiatives Office, 950 N. Cherry Ave., Tucson, AZ, USA 85719.

Second Bäckaskog Workshop on Extremely Large Telescopes, edited by Arne L. Ardeberg, 
design of the sensors, for virtually all of the existing designs it results in significant performance loss in controlling these low order modes. It was suggested ${ }^{5}$ to use optical detection, via the wavefront of a guide star, to measure these low order shape aberrations. The same - or a similar - optical sensor could also drive the rigid body motion of the secondary mirror to correct for larger scale structural deformations due to the wind load ${ }^{7}$.

Theoretical calculations have shown that an optical control loop, which is intended to correct wind induced aberrations by shaping the primary mirror, has the potential to introduce additional shape errors due to the anisoplanatism of atmospheric turbulence ${ }^{8}$.

It is essential to determine if we need optical feedback to mitigate wind buffeting, in addition to the mechanical feedback loops of edge sensors. In order to settle the question, we have to investigate the amount of energy channeled from wind into the unobservable primary mirror modes. Furthermore, all the other possible mechanisms degrading the image quality due to wind induced telescope deformations must be accounted for.

\section{THE INTEGRATED TELESCOPE MODEL}

To investigate the performance degradation of a 30-meter class telescope under wind buffeting, an integrated, dynamic model was developed. The model is based on the optical and structural point design of the Giant Segmented Mirror Telescope $\left(\right.$ GSMT) ${ }^{12}$ developed by the New Initiatives Office of AURA (Figure 3). AURA recently entered into a partnership with $\mathrm{CELT}^{9}$ and $\mathrm{HIA}^{10}$ (Canada) to develop conceptual and preliminary designs for a Thirty Meter Telescope, which will most likely be different from the model presented here.

The integrated model used for simulations of the wind effects is described in details elsewhere". It is based on two "virtual telescopes", independently assembled by the same algorithm in different software environments. The structural virtual telescope is built in MATLAB ${ }^{\circledR}$ as a set of differential equations. The coefficients of the equations as the eigenvalues and eigenvectors of the structure are developed in a finite element analysis (FEA) software tool, like I-DEAS, and are then imported into MATLAB.

The optical model, as another virtual copy of the same telescope, is built in C as user-defined surfaces and custom ray trace algorithms. The compiled $\mathrm{C}$ code is linked to the ray trace software, in our case CODE-V, as a dynamic link library (DLL) file. To synchronize the optical virtual telescope with the structural model, changes are made to the definition of the userdefined surfaces.

The telescope mount, primary mirror support truss and secondary mirror support tripod is modeled as flexible, dynamic structure with $2 \%$ damping ${ }^{12}$. The 618 segments of the primary mirror are grouped into rafts that hold seven segments each. The rafts are considered as rigid bodies, meaning their resonant frequencies fall above the range investigated in this paper. Each raft is connected to the support truss at a single node capable of transferring forces and torques. Each segment is supported on 3 actuators with stiffness of $10 \mathrm{~N} / \mu \mathrm{m}$ to facilitate tip, tilt and piston motion.

The resonances of the individual segments are also significantly higher than the bandwidth of wind forces, so the segment tip/tilt/piston displacements are not incorporated into the dynamic state space representation of the structure but rather considered as feed through ${ }^{11}$. This approach allows a modal reduction down to 100 modes and consequently speeds up the simulation.

The optical model supports tip, tilt, and piston displacements for each segment, as well as x decenter, y decenter, tip, tilt, and piston rigid body motion for the secondary mirror. The ray trace uses a 512 by 512 ray bundle, which corresponds to about 6 $\mathrm{cm}$ sampling resolution on the primary mirror.

The GSMT primary mirror features 3516 edge sensors, two on each inter-segment edge at the vertex points. The influence function $\mathbf{G}$ of the primary mirror is defined as the transformation from actuator commands a to edge sensor readings $\mathbf{s}$.

$$
\mathbf{s}=\mathbf{G a}=\mathbf{U} \mathbf{\Sigma} \mathbf{V}^{\top} \mathbf{a}
$$


The singular value decomposition of the influence function in Equation (3.1) defines actuator $\left(\mathbf{a}=\mathbf{V} \mathbf{a}_{m}\right)$ and sensor ( $\mathbf{s}=\mathbf{U} \mathbf{s}_{m}$ ) modes for the primary mirror. These modes are linked together through the singular values $\sigma_{i}$ of the influence function ${ }^{4}$.

$$
s_{m i}=\sigma_{i} a_{m i}
$$

As it's expected from the geometry, the singular values corresponding to the rigid body motion (tip, tilt, piston) of the primary mirror are practically zeros. Since the edge sensors assumed in the model cannot sense dihedral angle, a fourth mode, the focus mode is also unobservable. These unobservable modes are shown in Figure 2. It is worth to note that these shapes are indeed the linear combinations of the first four fringe Zernike terms, with clearly negligible higher order content.

The rest of the singular values - the observable ones - are shown in Figure 1. All the primary mirror modes defined by the singular values, including the unobservable ones, are normalized to the RMS value of $2.3 * 10^{-2}$.
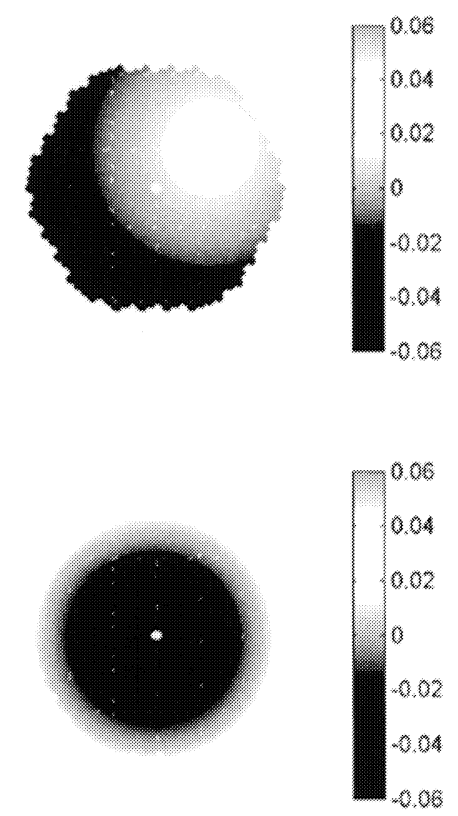

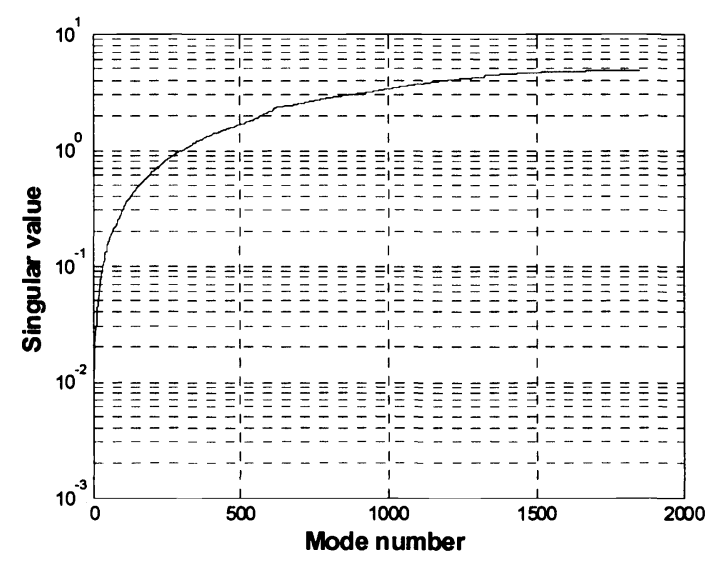

Figure 1 The non-zero singular values of the primary mirror influence function
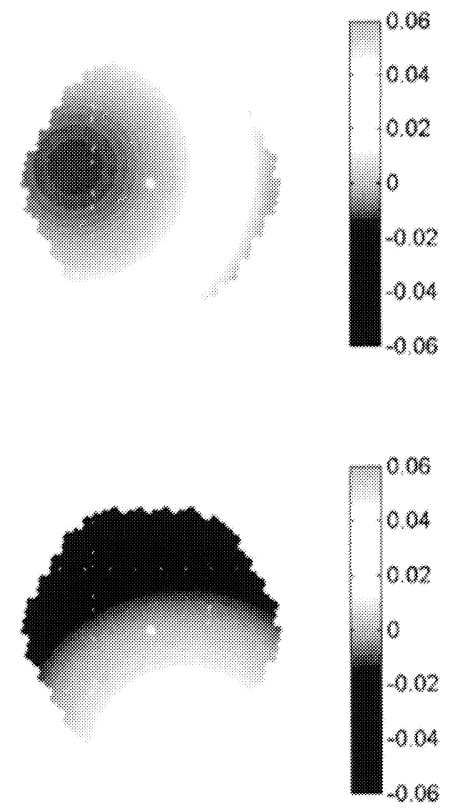

Figure 2 The first 4 actuator modes of the primary mirror that are not observable by the edge sensors 


\section{DISTURBANCE MODELS USED IN THE SIMULATION}

\subsection{Wind}

There are two distinct ways to apply wind forces to the telescope in our model. For the primary mirror, a pressure screen was developed and transformed into forces and momentums acting on individual segments. For the top end of the telescope, wind velocities with magnitudes and directions were assumed and transformed into drag forces.

In our comprehensive experiment at Gemini South ${ }^{13}$ we found that the air turbulence just above the primary mirror can be approximated as well-developed Kolmogorov turbulence ${ }^{12}$, except some transient areas around the edges and behind the baffle.

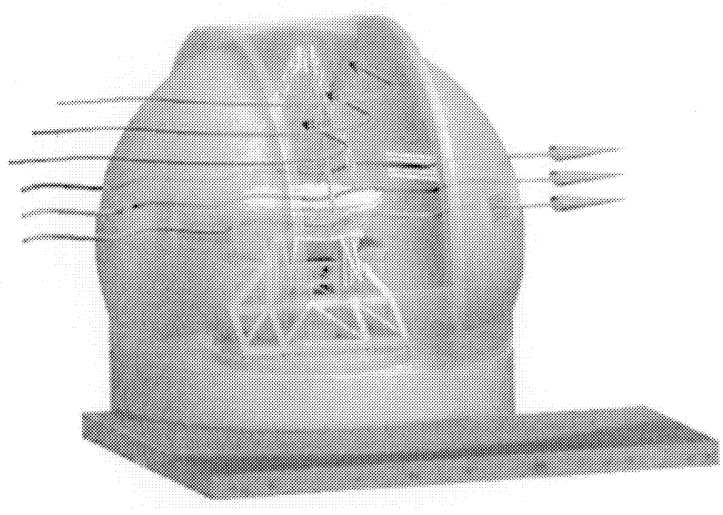

Figure 3 The assumed wind conditions for the simulations

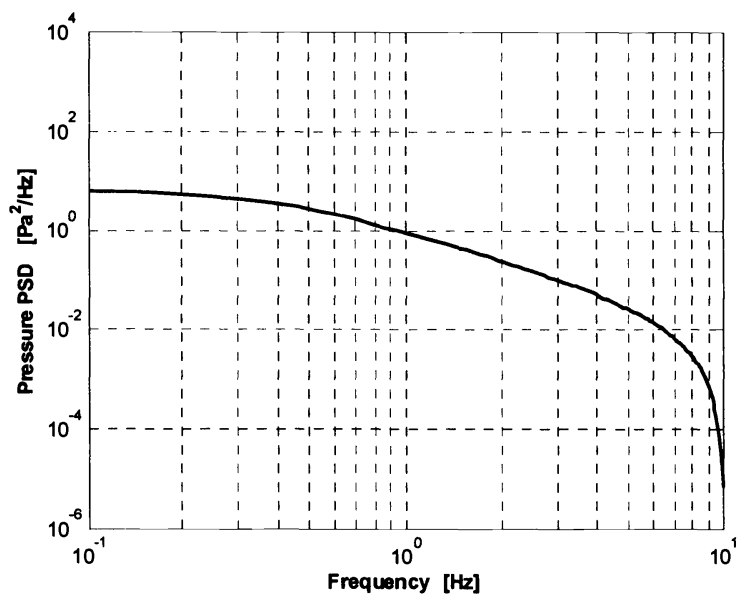

Figure 4 The average power spectral density of the pressure screen applied to the primary mirror

For our current simulation, we assume well-developed turbulence with a bandwidth of $0.4 \mathrm{~Hz}$ and RMS (zero mean) pressure of 2.5 $\mathrm{Pa}$. The mean wind pressure on the primary mirror constitutes a static perturbation, consequently it is outside of the scope of this paper. The bandwidth chosen corresponds to about 8.5 meters outer scale and 1 meter correlation length, assuming $3.5 \mathrm{~m} / \mathrm{s}$ mean wind velocity at the primary mirror ${ }^{14}$. These estimates are consistent with the measurements at Gemini South and our former conclusion that the outer scale at the mirror is most likely determined by the height of the wind gates and not by the mirror size ${ }^{12}$. The $3.5 \mathrm{~m} / \mathrm{s}$ is very close to the maximum allowable wind speed $(3.2 \mathrm{~m} / \mathrm{s})$ for Gemini ${ }^{15}$.

The pressure screen for our simulation was defined on a $1 \mathrm{~m}$ grid, as filtered, independent random series. The filter used is a first order low-pass filter with bandwidth of $0.4 \mathrm{~Hz}$ and gain of 8 . The resulted pressure series have slightly more power at higher frequency than the wind, because the Kolmogorov pressure spectrum has a steeper roll-off $\left(\mathrm{f}^{-7 / 3}\right.$, as shown in Figure 4). The extreme roll-off close to $10 \mathrm{~Hz}$ is an artifact of the digital filter due to the bilinear transformation used in its design.

The estimation of the drag forces on the top end of the telescope was also based on our measurements at Gemini South. In the same experiment used above for primary mirror pressure estimates ${ }^{12}$, the mean wind speed around the secondary mirror was found to be about $5 \mathrm{~m} / \mathrm{s}$. In the simulation, the actual wind velocity time series measured by the anemometer at the Gemini secondary mirror was used.

The drag forces $F_{D}$ can be calculated from the wind velocity $v$.

$$
\vec{F}_{D}=C_{D}\left(\frac{1}{2} \rho A\right)|v| \vec{v}
$$


Here $C_{D}, \rho$, and $A$ are the drag coefficient, air density and effective cross section of the structural element, respectively. We assumed a 2 meter high cylinder with 2 meter diameter as the secondary structure, with drag coefficient of 0.5 .

Since the simulation did not take into account the wind forces on the secondary support legs (tripod), the structural deformations reported are most likely underestimated. The lack of wind forces on the tripod is based on the assumption that the wind correlation length along the legs is short enough, so the integral effect of wind is negligible. This assumption is probably an oversimplification. Unfortunately, there is no available simulation or test data on the wind velocity distribution and its correlation length around the tripod.

\subsection{Atmosphere}

A ground layer Kolmogorov phase screen was generated using MATLAB. The parameters for the phase screen were, a Fried diameter $\left(r_{0}\right)$ of $20 \mathrm{~cm}$ at $0.5 \mu \mathrm{m}$ wavelength, and an infinite outer scale. The wind velocity expected was $5 \mathrm{~m} / \mathrm{s}$. The spatial sampling rate of the phase screen was $12 \mathrm{~cm}$, while the temporal one was set to $20 \mathrm{~Hz}$.

\section{SIMULATION RESULTS}

\subsection{Wind energy in unobservable modes}

Figure 5 indicates that most of the wind energy is concentrated into the lower order mirror modes. It also indicates the significance of wind buffeting on the secondary structure, especially for the lower order modes. The effect is even more visible, if we zoom into the lower end of the graph (Figure 6). For the first four modes, the difference is about four fold. The total RMS deformation in these modes due to full wind is about $3 \mu \mathrm{m}$. It is certainly not completely negligible, but even if we assume the unrealistic worst case that all of this deformation is in tilt, the resulting image jitter is less than 0.1 arcsec.

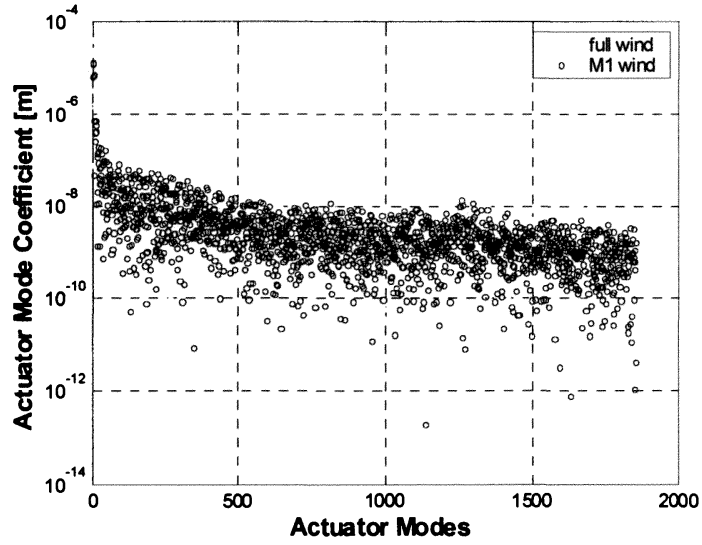

Figure 5 The actuator mode coefficients describing the primary mirror deformation due to wind forces on primary mirror only (M1 wind), and on both primary mirror and telescope top end (full wind)

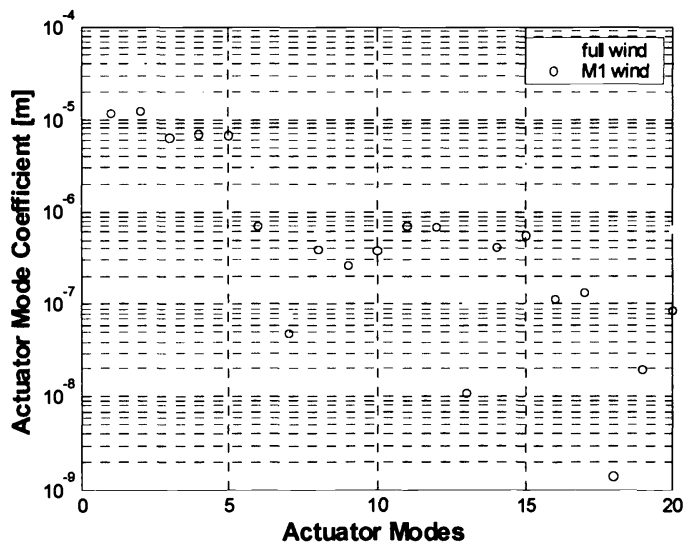

Figure 6 The first 20 actuator mode coefficients describing the primary mirror deformation due to wind forces on primary mirror only (M1 wind), and on both primary mirror and telescope top end (full wind)

\subsection{Wind effects on primary mirror continuity}

Considering the time history of each edge sensor, it can be characterized by its power spectral density. The average of these PSDs for all sensors (3516) is shown in Figure 7. As expected, with wind is applied only on the primary mirror, it nicely follows the average PSD of the wind pressure (Figure 4). However, if we apply wind forces on the top end of the telescope, the structural resonances show up in the edge sensor readings. Since the lowest order resonances are associated with 
"rocking" type motion of the telescope, the next set of resonances - associated with higher order support truss deformations has more pronounced effect on the continuity of the mirror.

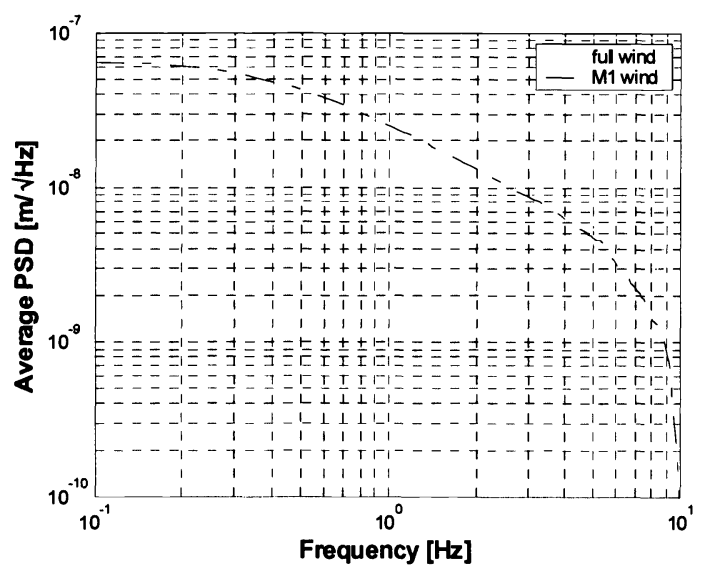

Figure 7 The average power spectrum density of the 3516 edge sensor displacements due to wind forces on primary mirror only (M1 wind), and on both primary mirror and telescope top end (full wind)

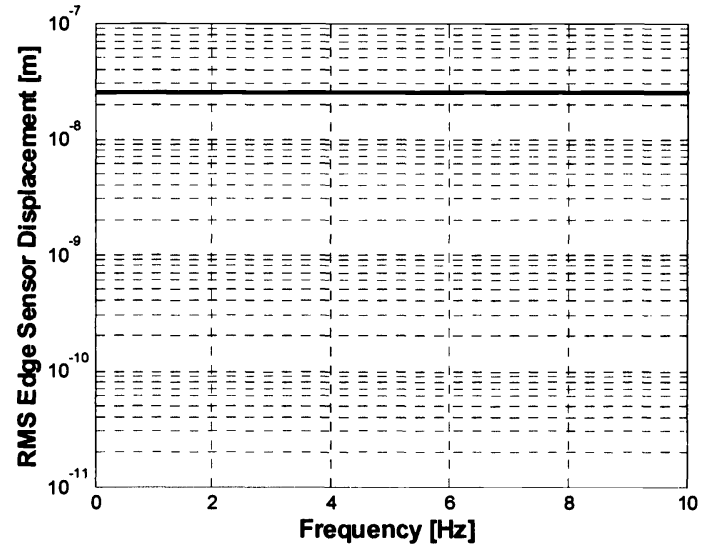

Figure 8 The RMS of the average edge sensor displacements atabove given frequencies, in case of full wind load. The solid line corresponds to $25 \mathrm{~nm}$ (error budget)

By definition, the integral of the PSD provides the RMS value of the time series. By integrating Figure 7 from a given frequency to infinity, we can estimate the RMS contribution of the signal residual at/above that frequency. This RMS residual can also be perceived as an error estimate for a feedback loop with control bandwidth equal to that given frequency (Figure 8). As it's shown in the figure, a $\sim 1 \mathrm{~Hz}$ bandwidth controller can limit the error to about $25 \mathrm{~nm}$, which is the value allocated in the preliminary error budget for GSMT.

\subsection{Optical effects of secondary mirror displacements}

We found - similarly to earlier investigations using less sophisticated models ${ }^{5}$, - that the wind buffeting on the top end of the telescope has the most pronounced effect on the exit pupil OPD.
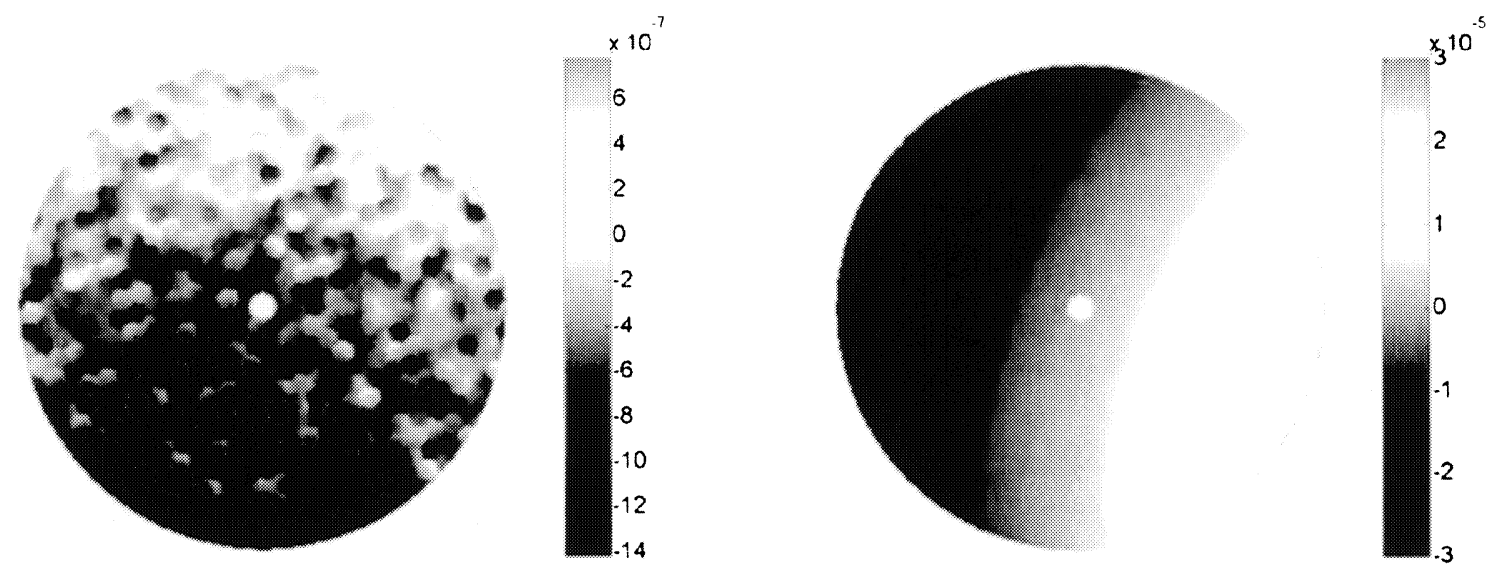

Figure 9 Snapshots of the exit pupil aberrations (OPD in meters) due to wind forces on primary mirror only (left), and on both primary mirror and telescope top end (right). (Note the different scale) 
Figure 9 shows two snapshots of the exit pupil OPD at the same time instant, with and without applying wind on the secondary structure. While the segmentation of the primary mirror is clearly visible in the left OPD map, applying wind on the top end of the telescope generates so much tilt that it covers up all other effects.

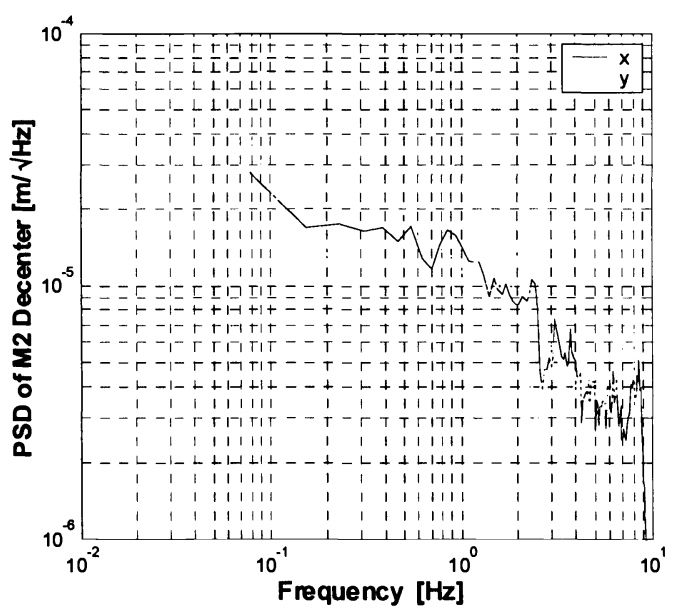

Figure 10 The power spectral density of secondary mirror de-center due to wind buffeting

The major cause of this tilt is the decenter of the secondary mirror, as seen in Figure 10. The relatively large amplitude of the displacements is combined with substantial bandwidth (above $1 \mathrm{~Hz}$ ), making the outcome more severe. Figure 11 shows the RMS Zernike decomposition of the exit pupil OPD over a 100 seconds period. It confirms that although the direction of the OPD tilt is changing by time, the largest contributor to image aberration is indeed tilt.

It is clearly visible in Figure 9 that, even without applying wind on the secondary mirror structure, the major aberration is still tilt. Figure 12 compares the PSD of this tilt to that of the much larger tilt caused by secondary mirror decenter. Besides the amount of tilt due to the two distinct subset of structural deformations, there is a noticeable bandwidth difference: the slope of the M1 tilt contribution is steeper.

To establish the severity of the overall (full wind) wind seeing, we compared it to the atmospheric seeing. Although the image jitter due to wind seeing has significantly larger bandwidth than the atmospheric tilt (Figure 13), the overall effect of atmosphere on the $80 \%$ encircled energy is still larger (Figure 14).

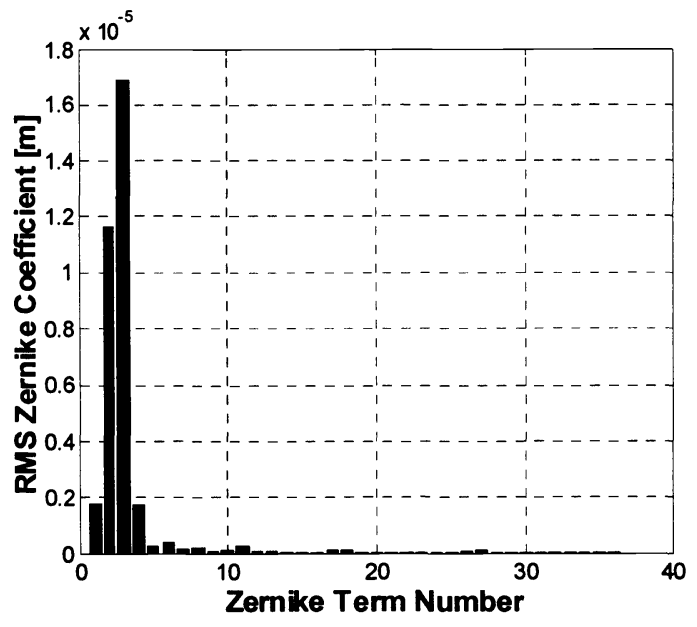

Figure 11 The RMS Zernike expansion of the exit pupil OPD

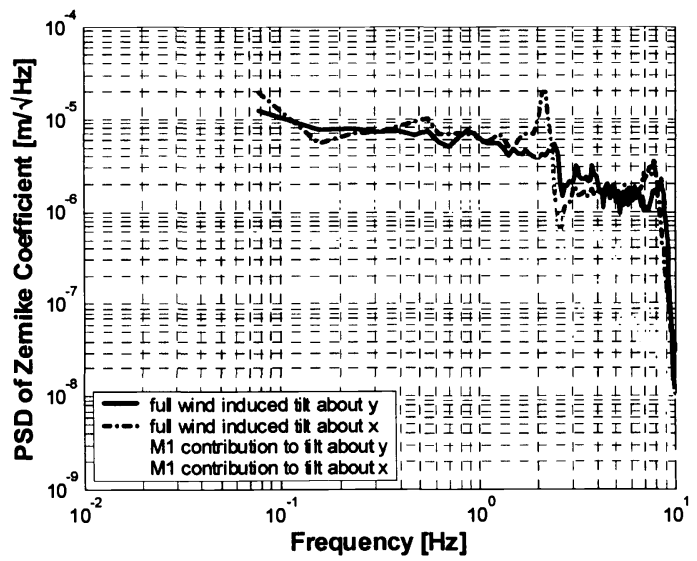

Figure 12 The contribution of primary mirror motion to the exit pupil OPD tip/tilt. Full wind contains wind on both primary mirror end top end 


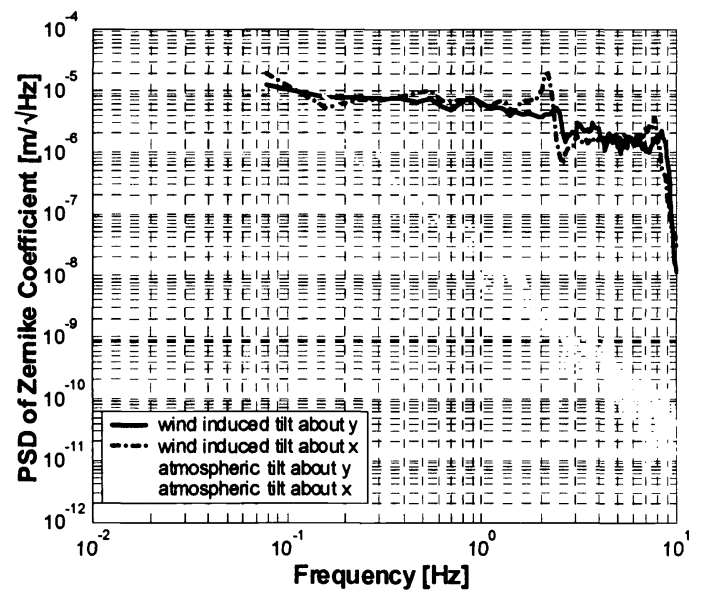

Figure 13 Comparison of wind and atmospheric seeing effects in exit pupil OPD

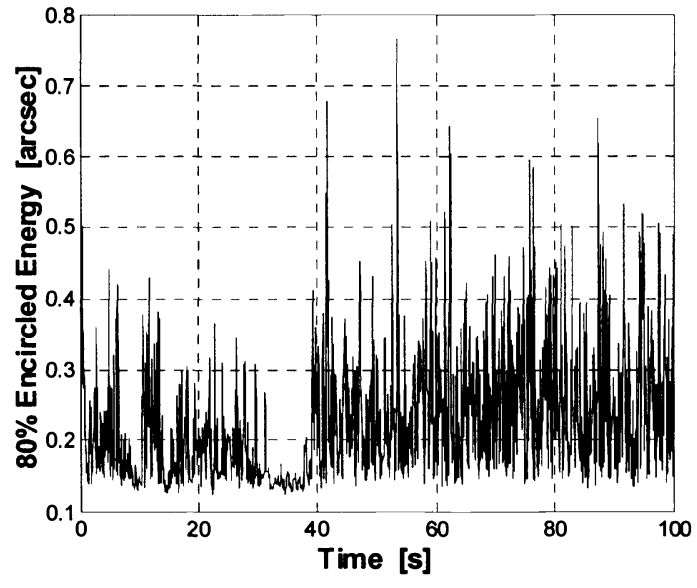

Figure 14 The $80 \%$ encircled energy due to wind seeing. In comparison, $20 \mathrm{~cm}$ Fried diameter at 0.5 $\mu \mathrm{m}$ wavelength corresponds to 0.5 arcsec seeing.

\section{CONCLUSION}

The large-scale structural deformations have limited impact on the continuity of the primary mirror. The major source of discontinuity is the direct wind pressure on the mirror. However, even assuming a worst case scenario when the correlation length of the wind pressure is very short (1 meter) and the RMS pressure variation is relatively large ( $2.5 \mathrm{~Pa})$, a control bandwidth of about $1 \mathrm{~Hz}$ seems to be adequate to keep the RMS step size below $25 \mathrm{~nm}$.

The wind seeing is dominated by image jitter - exit pupil OPD tip/tilt - mainly due to the decenter of the secondary mirror. However, in our simulations the wind seeing was smaller than the atmospheric seeing. This indicates that an optical feedback to control low order image aberrations may not be necessary in the seeing limited, wide field mode of the telescope.

It should be noted that the wind model used in the simulation based on assumptions not completely validated. The wind measurements at Gemini South provided quite detailed information about the pressure distribution on the dummy primary mirror, but they lacked any detail on wind velocities around the telescope structure. Since one of the most important concerns on the feasibility of extremely large telescopes is the severity of the wind load and its effect on the image quality, further investigation of the wind around the telescope structure is required. The major issues to be investigated are: (i) the interactions between the structure and wind velocity around the tripod supporting the secondary mirror; and in particular (ii) the spatial and temporal stochastic characteristics of the wind velocity and direction.

\section{ACKNOWLEDGEMENTS}

The New Initiatives Office is a partnership between two divisions of the Association of Universities for Research in Astronomy (AURA), Inc.: the National Optical Astronomy Observatory (NOAO) and the Gemini Observatory. NOAO is operated by AURA under cooperative agreement with the National Science Foundation (NSF). The Gemini Observatory is operated by AURA under a cooperative agreement with the NSF on behalf of the Gemini partnership: the National Science Foundation (United States), the Particle Physics and Astronomy Research Council (United Kingdom), the National Research Council (Canada), CONICYT (Chile), the Australian Research Council (Australia), CNPq (Brazil) and CONICET (Argentina). 


\section{REFERENCES}

1. T. Andersen, M. Owner-Petersen, H. Riewaldt, “An Integrated Simulation Model of the Euro50," Proceedings of SPIE 4757, 84-92, (2002).

2. T. S. Mast, J. E. Nelson, "Segmented mirror control system hardware for CELT", Proceedings of SPIE 4003, 226-240, (2000).

3. D. G. MacMartin, T. S. Mast, G. Chanan, J. E. Nelson, "Active Control Issues for the California Extremely Large Telescope," AIAA 2001-4035 (2001)

4. D. G. MacMartin, G. Chanan, "Control of the California Extremely Large Telescope," Proceedings of SPIE 4840, 6980 (2003).

5. G. Z. Angeli, M. K. Cho, M. S. Whorton, "Active optics and control architecture for a Giant Segmented Mirror Telescope," Proceedings of SPIE 4840, 129-139 (2003).

6. M. Troy, G. Chanan, E. Sirko, E. Lefert, "Residual Misalignments of the Keck Telescope Primary Mirror Segments: Classification of Modes and Implications for Adaptive Optics," Proceedings of SPIE 3352, 307-317 (1998).

7. M. Whorton, G. Z. Angeli, "Modern Control of the Secondary Mirror of a Giant Segmented Mirror Telescope," Proceedings of SPIE 4840, 140-150 (2003).

8. S. Padin, "Wind-induced deformations in a segmented mirror", Applied Optics, 41, No.13. 2381-2389 (2002).

9. The California Extremely Large Telescope, http://celt.ucolick.org/

10. VLOT -Very Large Optical Telescope, http://www.hia-iha.nrc-cnrc.gc.ca/projects/VLOT_e.html

11. G. Z. Angeli, A. Segurson, R. Upton, and B. Gregory, "Integrated modeling tools for large ground based optical telescopes," Proceedings of SPIE 5178-9 (2003). (In press)

12. Enabling a Giant Segmented Mirror Telescope for the Astronomical Community, http://www.auranio.edu/book/index.html, AURA New Initiatives Office, 2002

13. M. K. Cho, L.Stepp, G. Z. Angeli, D. Smith, "Wind loading of large telescopes," Proceedings of SPIE 4837, $352-367$ (2003)

14. G. Z. Angeli, M. K. Cho, M. Sheehan, L.Stepp, "Characterization of wind loading of telescopes," Proceedings of SPIE 4757, $72-83$ (2002)

15. M. K. Cho, L.Stepp, S. Kim, "Wind buffeting effects on the Gemini 8m primary mirrors," Proceedings of SPIE 4444, 302-314 (2001) 